Journal of the Mathematical Society of Japan Vol. 3, No. 1, May, 1951.

\title{
Sur une Généralisation de la Coupure de Dedekind
}

\section{Kinjiro KUnUguI}

1. En généralisant la notion de la coupure introduite par R. Dedekind, M. MacNeille ${ }^{1)}$ a donné une méthode d'obtenir une structure complèteq) à partir d'un ensemble quelconque (partiellement) ordonné.s) M. MacNeille a opéré comme il suit: étant donné un sous-ensemble $A$ d'un ensemble ordonné $R$ quelconque, on désigne par $A^{*}$ l'ensemble de tous les éléments de $R$ qui sont des bornes supérieures de $A$. De même, on désigne par $A_{*}$ l'énsemble de tous les éléments de $R$ qui sont des bornes inférieures de $A$ : Une paire des deux sous-ensembles $A_{1}, A_{2}$ de $R$ s'appelle une coupure, si l'on a, à la fois,

$$
A_{1}=\left(A_{2}\right)_{*}, \quad A_{2}=\left(A_{1}\right)^{*}
$$

et cette coupure au sens de MacNeille sera désignée par $\alpha=\left[A_{1}, A_{2}\right]$. L'ensemble de toutes les coupures, soit désigné par $\Re$, est ordonné par la définition suivante: on dit que deux coupures $\alpha^{\prime}=\left[A_{1}^{\prime}, A_{2}^{\prime}\right], \alpha^{\prime \prime}=\left[A_{1}^{\prime \prime}, A_{2}^{\prime \prime}\right]$ satisfont à la relation $\alpha^{\prime} \leq u^{\prime \prime}$ lorsqu'on a $A_{1}^{\prime} \subseteq A_{1}^{\prime \prime}$ (et cela revient à dire qu'on a $\left.A_{2}^{\prime} \supseteq A_{2}^{\prime \prime}\right)$. Avec cette définition de la coupure et de l'ordre des coupures, on voit facilement que l'ensemble $\mathfrak{R}$ est une structure complète. D'autre part, pour tout élément $a$ de $R$, l'ensemble $A_{1}(a)$ de tous les éléments $x$ de $R$ qui satisfont à $x \leq a$, et l'ensemble $A_{2}(a)$ de tous les éléments $y$ de $R$ qui satisfont à $y \geq a$ forment une coupure $\left[A_{1}(a), A_{2}(a)\right]$. Cette coupure peut être considérée come identique à l'élément $a$, et ainsi l'ensemble $R$ sera contenu dans $\mathfrak{R}$.

La notion de la coupure ainsi définie est une belle généralisation de la coupure employée par $\mathrm{R}$. Dedekind dans sa fameuse théorie des nombres irrationnels, et lorsqu'on l'applique à l'ensemble de tous les nombres rationnels, on obtient comme $\mathfrak{R}$ l'ensemble de tous les nombres iéels avec deux infinis $\pm \infty$.

2. Considérons maintenant la notion de la continuité au sens d'ordre. Pour cela, introduisons d'abord deux notions : la densité et la lacune. Un

1) MacNeille: Partially ordered sets, Trans. Am. Math. Soc., 42 (1937), pp. 416-460.

2) Voir p. ex. G. Birkhoff, Lattice theory, Am. Math. Soc. Colloquium publications Vol. XXV, New York City. 1940, p. 27.

3) Pour simplicité, nous appelons ensembłe ọ̣donnẹ tout ensemble qui est partiellemẹt ordonrê. 
élément $a$ d'un ensemblè ordonné $R$ s'appelle isolé au sense a'ordre (ou $o$ isolé) s'il n'est comparable avèc aucun élément de $R$ (sauf luimême). Un sous-ensemble $A$ d'un ensemble ordonné $R$ sera appelé dense dans $R$, si $A$ contient tous les éléments $o$-isolés de $R$ et si, avec tous les deux elements $a_{1} ; a_{2}$ de $R$ tels que $a_{1}<a_{2}{ }^{4)}$ il existe un élément $a_{3}$ de $A$ tel qu'ón ait $a_{1} \leq a_{3} \leq a_{2}$. Si, avec tous les deux elements $a_{1}, a_{2}$ de $R$ tols que $a_{1}<a_{2}$, il existe un element $a_{3}$ de $R$ tel qu'on ait $a_{1}<a_{3}<a_{2}$, nous disons simplement que $R$ est dense. Une coupure sera dite qu'elle forme une lacune, lorsque $A_{1}$ n'a pas un élément maximum (et cela revient à dire que $A_{2}$ n'a pas un élément minimum) et que ni $A_{1}$ ni $A_{2}$ n'est vide. Un ensemble ordonné $R$ est applelé continu au sens d'ordre (ou o-continu) si elle est dense et si l'ensemble $\mathfrak{R}$ de toues ses coupures n'a aucune lacune.

La question se pose alors si, pour tout ensemble ordonné $R$ qui est dense, l'ensemble $\mathfrak{R}$ des coupures de $R$, est-il un $o$-continu ? Mais, la réponse $\mathrm{y}$ est négative, comme nous pouvons en donner des exemples très simples. Fin effet, prenons comme $R$ l'ensemble des deux éléments $a, b$ et supposons que $a, b$ ne sont pas comparables. Alors l'ensemble $\mathfrak{R}$ des coupures de $R$ se compose des quatre éléments $O, a, b, 1$ tels qu'on ait $0=[O, R], 1=[R, O], 0<a, 0<b, a<1, b<1$. On voit bien que $R$ est dense. Mais $\Re$ ne l'est pas, puisqu'entre 0 et $a$ on n'a aucun élément de $\Re$.

Voici encore un autre exemple. Considéions deux ensembles de points $A_{1}$ et $A_{2}$ situés dans le plan, et définis comme il suit: $A_{1}$ est l'ensemble de tous les points $(x, y)$ qui satisfont à la relation : $x=0, y<0 . A_{2}$ est celui de tous les points $(x, y)$ satisfaisant à $y \geq 0, x=1$ ou -1 . Posons $R=A_{1}+A_{2}$. Deux points $\left(x_{1}, y_{1}\right),\left(x_{2}, y_{2}\right)$ de $R$ sont ordonné comme $\left(x_{1}, y_{1}\right)$ $<\left(x_{2}, y_{2}\right)$ lorsqu'on a $y_{1}<y_{2}$. On voit bien que $R$ est dense. Or, l'ensemble $\Re$ de toutes les coupures de $R$ se compose de $R$ et d'un élément $\alpha$ qui est la borne inférieure de $A_{2}$ et qui est la borne supérieure de $A_{1}$ en même temps. On a donc $u<(1,0)$, mais il n'existe aucune coupure de $R$ qui serait sitciée entre ces deux. Donc, $\mathfrak{R}$ n'est pas dense.

Disons qu'une définition de la coupure admet la proposition de Dedekind, si la définition nous permet de dire que l'ensemble $\mathfrak{R}$ de toues les coupures de $R$ est $o$-continu dès que $R$ soit dense.

3. Maintenant, nous allons donner une définition de coupure d'un ensemble ordonné quelconque telle qu'elle admet la proposition de Dedekind.

4) Nous désignoss par $a_{1}<a_{2}$, lorsqu'on a, à la fois, $a_{1} \leq a_{2}$ et $a_{1} \neq a_{2}$. 
Soit $R$ un ensemble ordonné quelconque. Considérons deux parties $A_{1}, A_{2}$ de $R$ qui satisfont aux quatre conditions suivantes:

$\left.1^{\circ}\right)$ On a $A_{1} \neq O, A_{2} \neq O$.

$\left.2^{\circ}\right)$ Si $a_{1} \in A_{1}, a_{2} \in A_{2}$ et $a_{1} \neq a_{2}$, on a $a_{1}<a_{2}$

$\left.3^{\circ}\right)$ Pour deux éléments quelconques $a_{1}, b_{1}$ de $A_{1}$, il existe au moins un élément $c_{1}$ de $A_{1}$ tel que $a_{1} \leq c_{1}, b_{1} \leq c_{1}$. Pour deux éléments quelconques $a_{2}, b_{2}$ de $A_{2}$, il existe au moins un élément $c_{2}$ de $A_{2}$ tel que $a_{2} \geq c_{2}, b_{2} \geq c_{2}$.

$\left.4^{\circ}\right) A_{1}, A_{2}$ sont des ensembles maxima parmi ceux qui satisfont aux conditioas $\left.\left.\left.1^{\circ}\right), 2^{\circ}\right), 3^{\circ}\right)$. En d'autres termes, si $A_{1}^{\prime} \supseteq A_{1}, A_{2}^{\prime} \supseteq \underline{D} A_{2}$ et si $A_{1}^{\prime}, A_{2}^{\prime}$ satisfont à $\left.\left.1^{\circ}\right), 2^{\circ}\right), 3^{\circ}$ ) (en remplaçant $A_{1}, A_{2} \operatorname{par} A_{1}^{\prime}, A_{2}^{\prime}$ respectivement) on a les identités : $A_{1}^{\prime}=A_{1}, A_{2}^{\prime}=A_{2}$.

Nous appelons cette paire $A_{1}, A_{2}$ une coupure de $R$ et la désignons par $u=\left(A_{1}, A_{2}\right)$. La définition de l'ordíe entre les coupures sera comme suivant : soient $\alpha=\left(A_{1}, A_{2}\right), \beta=\left(B_{1}, B_{2}\right)$ deux coupures. Nous posons par définition $\alpha=\beta$ lorsque nous'avons à la fois $A_{1}=B_{1}, A_{2}=B_{2}$. Nous posons $\alpha<\beta$ par définition lorsqu'il existe deux éléments $a_{2}, b_{1}$ tels que $a_{2} \in A_{2}$, $b_{1} \in B_{1}$ et $a_{2}<b_{1}$. Nous mettons enfin $\alpha \leq \beta$ au lieu d'avoir $\alpha=\beta$ ou $u<\beta$. Maintenant il est facile de voir que l'òrdre des coupures ainsi défir i satisfait à deux axiomes d'ordre:

1) Si $\alpha \leq \beta$ et $\beta \leq \mu$, on a toujours $\alpha=\beta$.

2) Si $\alpha \leq \beta$ ei si $\beta \leq \gamma$, on a toujours $\alpha \leq \gamma$.

Donc, l'ensemble $\mathfrak{R}$ de toutes les coupures de $R$ est ordonré.

Soit, maintenant, $a$ un élément quelconque de $R$. Déux ensembles $A_{1}(a), A_{2}(a)$ définis plus havt satisfont aux conditions $\left.\left.\left.1^{\circ}\right), 2^{\circ}\right), 3^{\circ}\right)$ et $\left.4^{\circ}\right)$. Donc, nous pouvons identifier la coupure $\left(A_{1}(a), A_{2}(a)\right)$ àvec $a$. On a alors $R \subseteq \Re$. De plus, avec cette définition d'identification et d'ordre de la coupure, nous pouvons établir facilement la proposition suivante :

3) Soit $a=\left(A_{1}, A_{2}\right)$ une coupure. Si $a_{1} \in A_{1}$ on a tonjours $a_{1} \leq u . \quad D e$ même, si $a_{2} \in A_{2}$ on a toujaurs $\alpha \leq a_{2}$.

4. Maintenant, nous allons montrer que notre coupure admet la proposition de Dedekind. Pour cela, montrons d'abord.

4) Pour tout ensemble ordonné $R, R$ est dense dans lensemble $\mathfrak{R}$ de toutes ses coupures. Si $R$ est dense, $\Re$ est également dense.

En effet, soit $a$ une coupure de $R$ qui est $o$-isolé dans $\Re$. Posons $a=\left(A_{1}, A_{2}\right)$. Or, diaprès la condition $\left.1^{\circ}\right)$, on a $A_{1} \neq O$. Il existe donc au moins un élément $a_{1}$ de $A_{1}$. Alors, en vertu de 3 ), on a $a_{1} \leq \alpha$, et puisque $\alpha$ est $o$-isolé, ceci veut dire $a_{1}=\alpha$. Donc, $\alpha \in R$. 
Soient ensuite $\alpha, \beta$ deux éléments de $\Re$ tels que $\alpha<\beta$ et posons. $\alpha=\left(A_{1}, A_{2}\right), \beta=\left(B_{1}, B_{2}\right)$. Nous avons alors, d'après la définition de l'ordre des coupures, deux éléments $a_{2}, b_{1}$ tels que $a_{2} \in A_{2}, b_{1} \in B_{1}$ et $a_{2}<b_{1}$. Or, en vertu du 3), nous avons $\alpha \leq a_{2}<b_{1} \leq \beta$. Donc, $R$ est dense dans $\Re$.

Si $R$ est dense, nous avons, pour telles coupures $\alpha, \beta$ et pour tels éléments $a_{2}, b_{1}$, un élément $c$ de $R$ tel que $a_{2}<c<b_{1}$. Par suite, d'après la définition de l'ordré, nous avons $\alpha<c<\beta$. Donc, $\mathfrak{R}$ est également dense.

Enfin, nous pouvons démontrer la proposition suivante:

5) Pour tout ensemble ordonné $R$, l'ensemble $\Re$ de toutes ses coupures n'a aucune lacune.

Démonstration. Supposons, par impossible, qu'il existe une coupure $\left(\mathfrak{a}_{1}, \mathfrak{a}_{2}\right)$ de $\mathfrak{R}$ telle que $\mathfrak{a}_{1} \cap \mathfrak{a}_{2}=O$. Désignons par $\alpha_{1}=\left(A_{1}^{\alpha_{1}}, A_{2}^{\alpha_{2}}\right)$ une coupure ce $R$ qui appartient à $\mathfrak{a}_{1}$ et $\alpha_{2}=\left(A_{1}^{\alpha_{2}}, A_{2}^{\alpha_{2}}\right)$ celle qui appartient à $\mathfrak{a}_{2}:$ Posons

$$
A_{1}=\underset{a_{1} \in \mathfrak{a}_{1}}{\cup} A_{1}^{a_{1}}, \quad A_{2}=\underset{\alpha_{2} \in \mathfrak{a}_{2}}{\cup} A_{2}^{\alpha_{2}}
$$

Puisque $\mathfrak{a}_{1} \neq O, \mathfrak{a}_{2} \neq O, A_{1}^{\alpha_{1}} \neq O, A_{2}^{\alpha_{2}} \neq O$, on a $A_{1} \neq O$ et $A_{2} \neq O$. Donc $A_{1}$, $A_{2}$ satisfont à la condition $1^{\circ}$ ).

Prenons ensuite deux éléments quelconques $a_{1}, a_{2}$ tels que $a_{1} \in A_{1}, \mathfrak{a}_{2} \in A_{2}$. Il existe alors $\alpha_{1}, \alpha_{2}$ de solte qu'on ait $a_{1} \in A_{1}^{\alpha_{1}}, a_{2} \epsilon A_{2}^{\alpha_{2}}$. Puisque $a_{1} \cap a_{2}=O$, nous avons $a_{1}<\mu_{2}$ et par suite il existe $u_{2}^{\prime}, \alpha_{1}^{\prime}$ tels que $a_{2}^{\prime} \in A_{2}^{\alpha_{2}}, a_{1}^{\prime} \in A_{1}^{\alpha_{2}}$ et $a_{2}^{\prime}<a_{1}^{\prime}$. Alors, les inégalités $a_{1} \leq a_{2}^{\prime}, a_{1}^{\prime} \leq a_{2}$ entrainent $a_{1}<a_{2}$. Donc, $A_{1}$, $A_{2}$ satisfont à la condition $2^{\circ}$ ).

Pour montrer que $A_{1}, A_{2}$ satisfont à la condition $3^{\circ}$ ), remarq ons d'abord que $\mathfrak{a}_{1} n^{\prime}$ a pas un élément maximum. ${ }^{\mathrm{s})}$ En effect é'ément maximum de $\mathfrak{a}_{1}$ est, d'après la condition $3^{\circ}$ ), l'élément de $\mathfrak{a}_{1}$ qui est le plus grand. Désigno:as-le par $u$. et posons $\mathfrak{a}_{2}^{\prime}=u \cup \mathfrak{a}_{2}$. La paire $\mathfrak{a}_{1}$ et $\mathfrak{a}_{2}^{\prime}$ satisfont aux conditions $\left.\left.1^{\circ}\right), 2^{\circ}\right), 3^{\circ}$ ). Donc, d'après $4^{\circ}$ ), on a $a_{2}=a_{2}^{\prime}$. Par suite $\alpha$ appartientrait à $\mathfrak{a}_{1} \cap \mathfrak{a}_{2}$. Ceci est contradictoire à la supposition: $\mathfrak{a}_{1} \cap \mathfrak{a}_{2}=O$.

Remarquons ensuite que, pour tout $\alpha_{1}$ de $\mathfrak{a}_{1}$, il existe un élément $a_{1}^{\prime}$ de

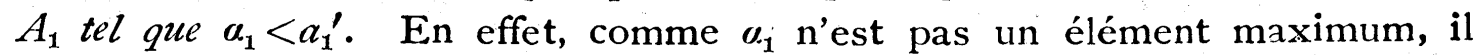
existe un élément $\alpha_{1}^{\prime}$ de $a_{1}$ tel que $\alpha_{1}<\alpha_{1}^{\prime}$. Posons $\alpha_{1}=\left(A_{1}^{\alpha_{1}}, A_{2}^{\alpha_{1}}\right), \alpha_{1}^{\prime}$ $=\left(A_{1}^{\alpha_{1}{ }^{\prime}}, A_{2}^{\alpha_{1}}\right)$. Puisque $\alpha_{1}<u_{2}^{\prime}$, il existe deux éléments $a_{2}, a_{1}^{\prime}$ tels que $a_{2} \in A_{2}^{\alpha_{1}}, \quad a_{1}^{\prime} \in A_{1}^{\alpha_{1}{ }^{\prime}}, \quad a_{2}<a_{1}^{\prime} . \quad$ D'autre part, nous avons, d'après 3$), a_{1} \leq a_{2}$. Donc, on a $a_{1}<a_{1}^{\prime}$. Or, $a_{1}{ }^{\prime} \epsilon A_{1}^{\alpha_{1}{ }^{\prime}}$ entraine $a_{1}{ }^{\prime} \in A_{1}$ et notre remarque est

5) Un element $e$ d'un ensemble ordonné $E$ s'appelle maximum si l'inégalité $e<x$ n'a lieu pour aucun $x$ de $E$. 


\section{démontrée.}

Revenons à la condition $3^{\circ}$ ). Soient $a_{1}, a_{1}^{\prime}$ deux éléments quelconques . de $A_{1}$. Il existe alors $a_{1}, a_{1}^{\prime}$ tels que $a_{1} \in A_{1}^{\alpha_{1}}, a_{1}^{\prime} \in A_{1}^{\alpha_{1}}, \alpha_{1} \in \mathfrak{a}_{1}, \alpha_{1}^{\prime} \in \mathfrak{a}_{1}$. Or, d'après la condition $\left.3^{\circ}\right)$ pour $\left(\mathfrak{a}_{1}, \mathfrak{a}_{2}\right)$, il existe un $\alpha_{1}^{\prime \prime}$ de $\mathfrak{a}_{1}$ tel que $u_{1} \leq \alpha_{1}^{\prime \prime}$, $\boldsymbol{\alpha}_{1}^{\prime} \leq \boldsymbol{\mu}_{1}^{\prime \prime}$. Comme nous avons remarqvé plus haut, il existe un élément $a_{1}^{\prime \prime \prime}$ de $A_{1}$ tel que $a_{1}^{\prime \prime}<a_{1}^{\prime \prime \prime}$. Donc, nous avons $a_{1} \leq a_{1} \leq a_{1}^{\prime \prime}<a_{1}^{\prime \prime \prime}$ et $a_{1}^{\prime} \leq \boldsymbol{\mu} \leq \boldsymbol{a}_{1}^{\prime \prime}<a_{1}^{\prime \prime \prime}$. Par conséquent, $A_{1}$ satisfait à la condiiion $\left.3^{\circ}\right)$. De même, $A_{2}$ satisfait à la même condition.

Ainsi, nous avons vu que $A_{1}, A_{2}$ satisfont à trois conditions $\left.1^{\circ}\right), 2^{\circ}$ ) et $3^{\circ}$ ). Alors, d'après le principe de Zorn, il existe deux ensembles $\bar{A}_{1}, \bar{A}_{1}$ tels que $\bar{A}_{1} \supseteq A_{1}, \bar{A}_{2} \supseteq A_{2}$ et qu'ils remplissent non seulement à trois conditions $\left.\left.\left.1^{\circ}\right), 2^{\circ}\right), 3^{\circ}\right)$ mais encore à $\left.4^{\circ}\right)$. $\left(\bar{A}_{1}, \bar{A}_{2}\right)$ forme donc une coupure de $R$. Posons $\alpha=\left(\bar{A}_{1}, \bar{A}_{2}\right)$. En vertu de l'inclusion $\bar{A}_{1} \supseteq A_{1}$ et de la proposition $3)$, nous pouvons dire que l'inégalité $a_{1} \leq \mu$ a lieu pour tout $a_{1}$ de $A_{1}$. Donc, d'après la remarque donrée plus haut (qui donne l'existence d'un élément $a_{1}$ tel que $\alpha_{1}<a_{1}$ ), on a $\alpha_{1}<\alpha$ pour tout $\alpha_{1}$ de $a_{1}$. De même $\alpha<\alpha_{2}$ a lieu pour tout $a_{2}$ de $a_{2}$. Ceci contredit évidemment à la condition $4^{\circ}$ ) de $\left(\mathfrak{a}_{1}, \mathfrak{a}_{2}\right)$. La proposition 5) est donc démontrée. 